\title{
Nonadiabatic neutrino oscillations reexamined
}

\author{
M. M. Guzzo, ${ }^{1,2}$ J. Bellandi, ${ }^{1}$ and V. M. Aquino ${ }^{1,3}$ \\ ${ }^{1}$ Instituto de Fisica "Gleb Wataghin," Universidade Estadual de Campinas, Unicamp 13083-970 Campinas, Sao Paulo, Brazil \\ ${ }^{2}$ Instituto de Fisica Teórica, Universidade Estadual Paulista, Unesp, Rua Pamplona, 145-01405 São Paulo, Sao Paulo, Brazil \\ ${ }^{3}$ Departamento de Fisica, Universidade Estadual de Londrina, 86000-970 Londrina, Paraná, Brazil
}

(Received 30 August 1993)

\begin{abstract}
Employing the Feynman procedure of ordered exponential operators and the stationary phase method to evaluate the multiple integrals involved, we calculate the level-crossing probability and analyze the role of a resonance in the evolution of a two-level neutrino system. We compare this procedure with more conventional ones, such as Landau's method and the ansatz of Kuo and Pantaleone and Petcov. We verify that our results reproduce the correct extreme nonadiabatic limit and give the standard solutions in the adiabatic regime for any arbitrary matter density distributions. We discuss in particular the case of solar neutrino propagation using the standard solar model predictions for the matter distribution in the Sun.
\end{abstract}

PACS number(s): $14.60 . \mathrm{Pq}, 96.60 . \mathrm{Kx}$

\section{INTRODUCTION}

Neutrinos propagating through matter oscillate in a different way than neutrinos propagating in vacuum $[1-4]$. This is because interactions in a medium modify the dispersion relations of particles traveling through. In quantum-mechanical language, a different dispersion relation signifies a different Hamiltonian of the system, which gives a different time evolution equation for the corresponding physical system.

Probably the most interesting consequence of the propagation in matter is the possibility of large neutrino mixing occurring when neutrinos cross a resonance region. Such an effect can be better appreciated observing the neutrino evolution equations in matter, which, for the two generation case, after neglecting irrelevant overall phases, can be written as [2]

$i \frac{d}{d t}\left(\begin{array}{l}v_{e} \\ v_{\mu}\end{array}\right)=\left(\begin{array}{cc}0 & \frac{\Delta}{4 E} \sin 2 \theta \\ \frac{\Delta}{4 E} \sin 2 \theta & \frac{\Delta}{2 E} \cos 2 \theta-\sqrt{2} G_{F} N_{e}(t)\end{array}\right)\left(\begin{array}{l}v_{e} \\ v_{\mu}\end{array}\right)$.

$\Delta=m_{2}^{2}-m_{1}^{2}$ is the squared mass difference of the vacuum mass eigenstates, $\theta$ is the neutrino mixing angle in vacuum, and $\sqrt{2} G_{F} N_{e}(t)$ is the consequence of electron neutrino coherent forward scattering from electrons in matter, the number density of which at the region reached by neutrinos at instant $t$ is $N_{e}(t)$. The physical eigenstates in matter, $v_{1}$ and $v_{2}$, are obtained from the current eigenstates $v_{e}$ and $v_{\mu}$ through a convenient twodimensional rotation which diagonalizes the $2 \times 2$ evolution matrix appearing in Eq. (1). Such a rotation can be parametrized by the mixing angle in matter $\widetilde{\theta}$ and one can write

$$
\begin{aligned}
& v_{1}(t)=v_{e}(t) \cos \widetilde{\theta}(t)-v_{\mu}(t) \sin \widetilde{\theta}(t), \\
& v_{2}(t)=v_{e}(t) \sin \widetilde{\theta}(t)+v_{\mu}(t) \cos \widetilde{\theta}(t),
\end{aligned}
$$

where $\widetilde{\theta}(t)$ is such that

$$
\sin ^{2} 2 \widetilde{\theta}(t)=\frac{\sin ^{2} 2 \theta}{\left[\frac{2 E \sqrt{2} G_{F} N_{e}(t)}{\Delta m^{2}}-\cos 2 \theta\right]^{2}+\sin ^{2} 2 \theta} .
$$

Thus $\widetilde{\theta}$ is substantially modified by the neutrino coherent scattering from the medium. If $N_{e}(t) \rightarrow 0, \widetilde{\theta} \rightarrow \theta$ and we recover vacuum expressions. When the brackets in the denominator of Eq. (3) vanish, a resonance occurs, $\widetilde{\theta} \rightarrow \pi / 4$ and the mixing between flavor eigenstates is maximal. Finally, when $N_{e}(t)$ is extremely large, $\widetilde{\theta} \rightarrow \pi / 2$.

It is well known that any physical information about the system of evolution equations (1) can be easily obtained if the following condition is satisfied: the difference of off-diagonal elements of the evolution matrix written in the matter physical eigenstate basis $\left(v_{1}, v_{2}\right)$ are negligible compared with the difference of diagonal elements of this same matrix. In this case, matter mass eigenstates propagate without mixing and one has the so-called adiabatic propagation. It can be verified [4] that this adiabaticity condition is hardest to satisfy at the resonance point. That is why one defines the adiabaticity parameter $\gamma$ [1], on which the evolution equations crucially depend, as the ratio between off-diagonal and diagonal entries of the matter mass eigenstates evolution matrix at the resonance point:

$$
\gamma \equiv \frac{\Delta}{E} \frac{\sin ^{2} 2 \theta}{\cos 2 \theta}\left|\frac{d}{d t} \ln N_{e}(t)\right|_{\mathrm{res}}^{-1} .
$$

When $\gamma \gg 1$, the propagation is adiabatic everywhere. Nevertheless, if $\gamma$ is of order 1 or smaller, off-diagonal and diagonal elements can be comparable and transitions between $v_{1}$ and $v_{2}$ or vice versa can occur.

We are typically interested in calculating quantities 
such as the averaged probability of finding an electronic neutrino at an instant $t$, if it was created at an instant $t_{0}$ as a given combination of $v_{1}$ and $v_{2}$ [5],

$P\left(v_{e} \rightarrow v_{e} ; t, t_{0}\right)=\frac{1}{2}\left[1+(1-2 P) \cos 2 \widetilde{\theta}\left(t_{0}\right) \cos 2 \widetilde{\theta}(t)\right]$,

where one has introduced the level-crossing probability of one matter mass eigenstate to be converted into another in the vicinity of a resonance region,

$$
P=\left|\left\langle v_{2}\left(t_{+}\right) \mid v_{1}\left(t_{-}\right)\right\rangle\right|^{2}
$$

and $t_{-}$and $t_{+}$refer to two far-away points on either side of the resonance, localized in regions where the propagation is adiabatic. Note that unitarity guarantees that $P$ is also the crossing probability for the $v_{2} \rightarrow v_{1}$ transition. One expects that for $\gamma \gg 1$, no level crossing occurs; i.e., the propagation is completely adiabatic and, consequently, $P=0$. In this particular case, one easily obtains all the relevant information about the evolution of the physical system just using adiabatic equations, such as Eq. (5) when $P=0$. Nevertheless, if $\gamma$ is of order 1 or smaller, $P \neq 0$ and one has to calculate it.

The purpose of this paper is to discuss an alternative way to calculate the level-crossing probability $P$. We use the Feynman procedure of ordered exponential operators [6-8] and the stationary phase method [9] to evaluate the multiple integrals involved, to write a solution for the system of equations (1) valid for any parametrization of the matter density distribution $N_{e}$. We compare the obtained result for $P$ with more conventional ones, such as the level-crossing probability from Landau's method and from the ansatz of Kuo and Pantaleone and Petcov to verify a rather accurate coincidence for a large range of the relevant physical parameters.

\section{CONVENTIONAL METHODS OF CALCULATING $P$}

The usual procedure to obtain the level-crossing probability $P$ consists in solving exactly the wave equations derived from Eq. (1). One uses the flavor eigenstates $v_{e}$ and $v_{\mu}$ to write two coupled first-order differential equations. It is possible then to decouple this system of equations eliminating one of the flavor eigenstates from these equations and obtaining a single second-order differential equation involving only the resulting flavor eigenstate.

Having the solution of the second-order equation, one can calculate, for instance, the electron neutrino survival probability. After making asymptotic approximations around the production and detection points and discarding nonclassical oscillating terms, the result, compared with Eq. (5), shows an expression for the level-crossing probability $P$.

Nevertheless, exact, analytic solutions of this secondorder differential equation are not easy to find in its most general form. It has been found that they exist for only a few functional forms of the matter density distribution: the linear [5,10-13], exponential [14-17], hyperbolic tangent [18], and $1 / r$ density distributions [19]. These density distributions are not always those that appear in nature. We can quote some examples. Supernova density distributions approach a $1 / r^{3}$ form, which cannot be satisfactorily described by any of the above distributions. For the solar case, an exponential function can fit the matter density distribution predicted by the standard solar model $[20,21]$ for a large range of the radial distance. But for the inner $15 \%$ of this distance, as well as for regions close to the solar surface this exponential fit does not work well.

The difficulty for obtaining general exact solutions of the evolution equation has motivated the search for alternative methods of calculating the level-crossing probability. In fact, nonadiabatic transition probability between two states was calculated some time ago [11-13] in the context of atomic physics. What is now called Landau's method was applied to the neutrino case $[5,10,12,19]$ to obtain a simple expression for $P$ :

$$
P=\exp (-\gamma F)
$$

where the quantity $F$ depends on how $N_{e}(t)$ varies near resonance. However, this equation gives only the leading exponential piece of the level-crossing probability, the dominant part for large $\gamma$. In the opposite case, when $\gamma$ is close to zero and the transition is extremely nonadiabatic, there may be other contributions to $P$. In fact, in Ref. [19], the extreme nonadiabatic limit for $P$ was evaluated:

$$
\lim _{\gamma \rightarrow 0} P=\cos ^{2} \theta
$$

Obviously, this indicates that, at least in this limit case, Landau's expression (7) fails. The same author developed then an ansatz for $P$ based on Landau's formula that avoids this problem $[19,22]$ :

$$
P=\frac{\exp (-\gamma F)-\exp \left(-\gamma F / \sin ^{2} \theta\right)}{1-\exp \left(-\gamma F / \sin ^{2} \theta\right)}
$$

For $\gamma$ greater than order $\sin ^{2} \theta$ this expression for $P$ is dominated by the first term in the numerator which coincides with Landau's expression, Eq. (7).

\section{THE FEYNMAN PROCEDURE OF ORDERED EXPONENTIAL OPERATORS}

To obtain the level-crossing probability $P$, the wave equations (1) have to be solved. We use the Feynman procedure of ordered exponential operators [6-8] to write a formula solution for any parametrization of the evolution matrix elements (1); in particular, for any arbitrary matter density distribution. Using Eqs. (6) and (2), it is easy to calculate the relevant level-crossing amplitude of probability around a resonance:

$$
\begin{aligned}
\left\langle v_{2}\left(t_{+}\right) \mid v_{1}\left(t_{-}\right)\right\rangle= & A_{R}\left(v_{e} \rightarrow v_{e}\right) \sin \widetilde{\theta}\left(t_{+}\right) \cos \widetilde{\theta}\left(t_{-}\right)-A_{R}\left(v_{\mu} \rightarrow v_{e}\right) \sin \widetilde{\theta}\left(t_{+}\right) \sin \widetilde{\theta}\left(t_{-}\right) \\
& +A_{R}\left(v_{e} \rightarrow v_{\mu}\right) \cos \widetilde{\theta}\left(t_{+}\right) \cos \widetilde{\theta}\left(t_{-}\right)-A_{R}\left(v_{\mu} \rightarrow v_{\mu}\right) \cos \widetilde{\theta}\left(t_{+}\right) \sin \widetilde{\theta}\left(t_{-}\right)
\end{aligned}
$$


where the subscript $R$ indicates transitions around resonance.

In order to evaluate the level-crossing probability $P$ we have to calculate the amplitudes of probability appearing in Eq. (10). Therefore, we want to obtain a solution of the system of equations (1) in a time interval $\left[t_{-}, t_{+}\right]$using an arbitrary boundary condition $\left(v_{e}\left(t_{-}\right), v_{\mu}\left(t_{-}\right)\right)$. Let us assume that electron and muon neutrino states are described by a two-component column spinor

$$
\phi(t)=\left(v_{e}(t), v_{\mu}(t)\right) \text {. }
$$

In order to simplify our expressions we will introduce a new spinor $\psi(t)$ related to $\phi(t)$ through the convenient phase transformation

$$
\begin{gathered}
\phi(t)=\exp \left[-\frac{i}{2} \int^{t} d t^{\prime}\left[\frac{\Delta}{2 E} \cos 2 \theta-\sqrt{2} G_{F} N_{e}\left(t^{\prime}\right)\right]\right. \\
\left.\times\left(1-\sigma_{3}\right)\right] \psi(t),
\end{gathered}
$$

where we have introduced the basis of the Pauli matrix $\left(\mathbb{1}, \sigma_{i}\right), i=1,2,3$. Since we are interested in transition amplitudes around the resonance, and the quantity

$$
\left(\Delta / 2 E \cos 2 \theta-\sqrt{2} G_{F} N_{e}\right)
$$

is negligible in this region [see Eq. (3)], the phase factor relating $\psi$ to $\phi$ reduces to just an irrelevant phase factor. Consequently, all the relevant information about the physical system we are interested in is totally given by $\psi(t)$. It can be shown, substituting Eq. (11) into (1), that

$i \frac{d}{d t} \psi(t)=\frac{\Delta}{4 E} \sin 2 \theta\left(e^{i \rho(t)} \sigma_{-}+e^{-i \rho(t)} \sigma_{+}\right) \psi(t)$.

$\sigma_{+}$and $\sigma_{-}$are the lowering and raising operators and

$$
\rho(t)=\int^{t} d t^{\prime}\left(\frac{\Delta}{2 E} \cos 2 \theta-\sqrt{2} G_{F} N_{e}\left(t^{\prime}\right)\right) .
$$

We can now apply the Feynman procedure to write a formal solution for Eq. (12):

$$
\begin{aligned}
\psi\left(t_{+}\right)= & \operatorname{Exp}\left[-i \int_{t_{-}}^{t_{+}} d t^{\prime} \frac{\Delta}{4 E} \sin 2 \theta\left(e^{i \rho\left(t^{\prime}\right)} \sigma_{-}\right.\right. \\
& \left.\left.+e^{-i \rho\left(t^{\prime}\right)} \sigma_{+}\right)\right] \\
& \times \psi\left(t_{-}\right),
\end{aligned}
$$

where Exp indicates an expansional defined as a sum of multiple ordered integrals [6-8].

If we now assume that $\rho(t)$ is large, as is the case for a large range of values of the physical interesting matter distributions $N_{e}(t)$, the integrand in Eq. (14) oscillates very quickly leading to vanishing contributions to the integration unless some stationary phase $t_{R}$ can be found in the interval of integration $t_{-}<t_{R}<t_{+}$. The value of the stationary phase is obtained through the relation [9]

$$
\left.\frac{d}{d t} \rho(t)\right|_{t=t_{R}}=0 \Longrightarrow \frac{\Delta}{2 E} \cos 2 \theta=\sqrt{2} G_{F} N_{e}\left(t_{R}\right) \text {. }
$$

Note that this stationary phase condition coincides with the resonance condition for which the mixing angle in matter $\widetilde{\theta}$, given by Eq. (3), is maximal. The major contribution to the value of the integrals appearing in Eq. (14) arises from the vicinity of the resonance point, i.e., the point where condition (15) is satisfied. This is the main result of the method of stationary phase and this is why we believe the Feynman procedure of ordered exponential operators together with the stationary phase method is indicated to analyze what happens around the resonance, even if the propagation in this region is in the nonadiabatic regime, which is, according to our previous discussion, difficult to analyze through other procedures.

Assuming that the condition (15) for the existence of a stationary phase is satisfied, i.e., there exists a resonance, we can write expressions for the amplitudes of the probability which enter in Eq. (10). Thus the amplitude of probability $A_{R}\left(v_{e} \rightarrow v_{\mu}\right)$ in a resonance region is simply given by the upper off-diagonal term of the $2 \times 2$ matrix of the Eq. (14) calculated through the stationary phase method [9]. The other relevant amplitudes $A_{R}\left(v_{e} \rightarrow v_{e}\right)$, $A_{R}\left(v_{\mu} \rightarrow v_{e}\right)$, and $A_{R}\left(v_{\mu} \rightarrow v_{\mu}\right)$ are given, respectively, by the upper diagonal, lower off-diagonal, and lower diagonal elements of the same matrix.

Let us now calculate the first-order contribution of these amplitudes. If we want to evaluate the $v_{e} \rightarrow v_{\mu}$ transition, terms accompanying $\sigma_{+}$in Eq. (14) have to be considered and we can write

$$
\begin{aligned}
A_{R}\left(v_{e} \rightarrow v_{\mu}\right)=- & i\left(2 \pi\left|\frac{d^{2} \rho(t)}{d t^{2}}\right|_{t=t_{R}}^{-1}\right)^{1 / 2} \frac{\Delta}{4 E} \sin 2 \theta \\
& \times \exp \left(i \rho\left(t_{R}\right)+i \frac{\pi}{4}\right) .
\end{aligned}
$$

We have applied the stationary phase method [9] to evaluate the relevant integral. Observe that this result is only valid if condition (15) is satisfied somewhere along the neutrino trajectory; otherwise $A_{R}\left(v_{e} \rightarrow v_{\mu}\right)$ is negligibly small.

Equation (16) is just a first-order approximation. It can be improved by analyzing the upper order terms in the expansion of the (14). It is not difficult to verify that contributions to $v_{e} \rightarrow v_{\mu}$ transitions come from terms of order odd in this expansion. Since we are assuming that we have only one stationary phase, the expansion in all orders can be calculated and we obtain

$$
\begin{gathered}
A_{R}\left(\nu_{e} \rightarrow v_{\mu}\right)=- \\
i 2 \xi\left(1-\xi^{2}+\xi^{4}-\xi^{6}+\cdots\right) \\
\times \exp \left(i \rho\left(t_{R}\right)+i \frac{\pi}{4}\right),
\end{gathered}
$$

where

$$
\xi=\left(\left.\frac{\pi}{2}\left|\frac{d^{2} \rho(t)}{d t^{2}}\right|_{t=t_{R}}^{-1}\right|^{1 / 2} \frac{\Delta}{4 E} \sin 2 \theta\right.
$$

For $\xi^{2}<1$, the series appearing in Eq. (17) is convergent [23] and the whole amplitude can be written as 


$$
A_{R}\left(v_{e} \rightarrow v_{\mu}\right)=-i \frac{2 \xi}{1+\xi^{2}} \exp \left(i \rho\left(t_{R}\right)+i \frac{\pi}{4}\right)
$$

In a completely analogous way we evaluate the opposite $v_{\mu} \rightarrow v_{e}$ transition amplitude

$$
A_{R}\left(v_{\mu} \rightarrow v_{e}\right)=-i \frac{2 \xi}{1+\xi^{2}} \exp \left(-i \rho\left(t_{R}\right)-i \frac{\pi}{4}\right),
$$

as well as the survival amplitudes of probability, given now by terms of even order in the expansional:

$$
A_{R}\left(v_{e} \rightarrow v_{e}\right)=A_{R}\left(v_{\mu} \rightarrow v_{\mu}\right)=\frac{1-\xi^{2}}{1+\xi^{2}} .
$$

Note that the electron and muon neutrino survival amplitudes are equal to each other while $v_{e} \rightarrow \nu_{\mu}$ and $v_{\mu} \rightarrow v_{e}$ transitions differ by one phase factor.

Finally we can put Eqs. (19)-(21) into (10) and (6) to obtain the final expression for the level-crossing probability:

$$
\begin{aligned}
P= & \left(\frac{1-\xi^{2}}{1+\xi^{2}}\right)^{2} \sin ^{2}\left[\widetilde{\theta}\left(t_{+}\right)-\widetilde{\theta}\left(t_{-}\right)\right] \\
+ & \frac{2 \xi^{2}}{\left(1+\xi^{2}\right)^{2}}\left\{\cos ^{2}\left[\widetilde{\theta}\left(t_{+}\right)-\widetilde{\theta}\left(t_{-}\right)\right]\right. \\
& \left.+\cos ^{2}\left[\widetilde{\theta}\left(t_{+}\right)+\widetilde{\theta}\left(t_{-}\right)\right]\right\},
\end{aligned}
$$

where we have again neglected oscillating terms. Supposing that the neutrino production and detection positions are far above and below the resonance region, respectively, it is natural to consider

$$
\widetilde{\theta}\left(t_{-}\right)=\pi / 2 \text { and } \widetilde{\theta}\left(t_{+}\right)=\theta .
$$

Furthermore, since the second derivative of $\rho(t)$, Eq. (13), is essentially the first derivative of the matter density distribution $N_{e}(t)$, we compare Eqs. (4) and (18) to conclude, after evaluating the involved quantities at the resonance point through Eq. (15), that

$$
\xi^{2}=\frac{\pi}{16} \gamma
$$

Interesting enough, we observe that in the extreme nonadiabatic limit, when $\gamma=(16 / \pi) \xi^{2} \rightarrow 0$, we recover from Eqs. (22) and (23) the correct limit for $P$ presented in Eq. (8), differently from what happens with Landau's expression (7). Without any ansatz, we have obtained the correct nonadiabatic limit.

This result is shown in Fig. 1 where we compare our expression for $P$, Eq. (22), with the usually accepted expression for this same quantity, Eq. (9). We assume an electron density distribution decreasing exponentially along the neutrino trajectory $[20,21]$,

$$
N_{e}(t)=2.4 \times 10^{26} e^{-t /(0.09 \text { solar radius })} \mathrm{cm}^{-3} \text {, }
$$

which is in good agreement with the standard solar model predictions for the matter density distribution inside the Sun, except for regions close to the solar center or to the solar surface and implies that $F$ appearing in Eq. (9) is $F=1-\tan ^{2} \theta[14-17]$.
We observe from Fig. 1 that our expression (22) and (23) for $P$ coincides with the conventional expression (9) for small values of $\gamma$, i.e., in the extreme nonadiabatic regime. For $\gamma$ of order 1, three cases have to be considered. For a large range of values of $\theta$ ( $\theta$ of order or smaller than 0.2), this agreement is rather accurate everywhere. For $\theta \approx 0.5$, a disagreement becomes appreciable in the region where $\gamma$ is around 1. Finally, for a maximal neutrino mixing angle in vacuum, $\theta \rightarrow \pi / 4$, both expressions (9) and (22) coincide again and are approximately equal to $\frac{1}{2}$ independently of $\gamma$. Obviously, this is not an interesting physical case and is usually discarded.

It should be noticed that the conventional expression (9) differs from the numerically calculated values of $P$ [16] in regions where $\gamma \approx 1$ and $\theta$ is large $(\theta \approx 0.4-0.6)$. We believe, furthermore, that our expression (22) does not have a strong dependence with $\theta$. Thus we expect the same accuracy of our expression for small or large $\theta$. Our main limitation comes from the lack of an expression for $P$ if $\xi^{2}=(\pi / 16) \gamma>1$. Nevertheless, this is exactly the

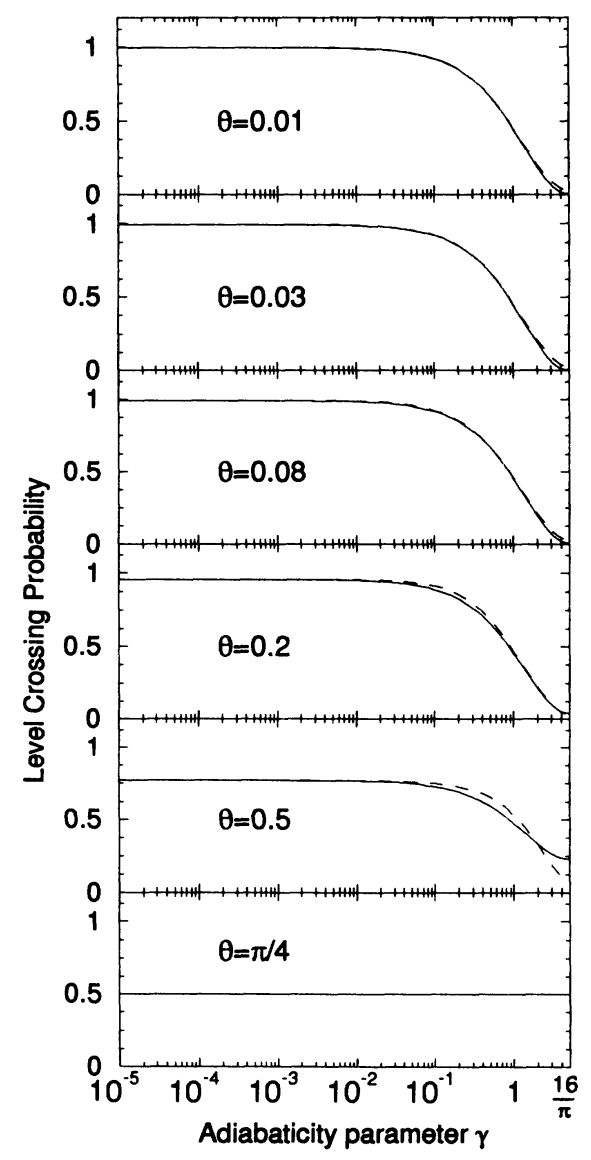

FIG. 1. Comparison of our expression for the level-crossing probability $P$, Eq. (22) (solid line), with the usually accepted expression for this same quantity, Eq. (9) (dashed line), as a function of the adiabaticity parameter $\gamma$, Eq. (4), for various values of the vacuum mixing angle $\theta$ and for $\xi^{2}=(\pi / 16) \gamma<1$. We have assumed that the electron number density varies exponentially according to Eq. (25) and taken $\widetilde{\theta}\left(t_{-}\right)=\pi / 2, \widetilde{\theta}\left(t_{+}\right)=\theta$. Note that for the limit case when $\theta \rightarrow \pi / 4$, both curves coincide presenting a constant value $\frac{1}{2}$, independently of $\gamma$. 
beginning of the adiabatic regime and we know that in this region $P$ goes quickly to zero.

\section{CONCLUSION}

Using the Feynman procedure of ordered exponential operators and the stationary phase method to solve the multiple integrals involved, we have analyzed the role of the resonance in a nonadiabatic neutrino propagation in a concise and general way. We have obtained an expression, Eq. (22), for the level-crossing probability valid for $\gamma$ of order or smaller than 1 , which reproduces the correct extreme nonadiabatic limit and coincides with the conventional expression for $P, \mathrm{Eq}$. (9), for a large range of the involved parameters.

\section{ACKNOWLEDGMENTS}

We would like to thank the Brazilian institutions CNPq, CAPES, and FAPESP for partial financial support.
[1] S. P. Mikheyev and A. Yu. Smirnov, Nuovo Cimento C 9, 17 (1986); Yad. Fiz. 42 (1985) [Sov. J. Nucl. Phys. 42, 913 (1985)].

[2] L. Wolfenstein, Phys. Rev. D 17, 2369 (1978); 20, 2634 (1979).

[3] H. Bethe, Phys. Rev. Lett. 56, 1305 (1986).

[4] S. M. Bilenky and S. T. Petcov, Rev. Mod. Phys. 59, 671 (1987).

[5] S. J. Parke, Phys. Rev. Lett. 57, 1275 (1986).

[6] R. P. Feynman, Phys. Rev. 84, 108 (1951); I. Fujikawa, Prog. Theor. Phys. 7, 433 (1952).

[7] J. Bellandi et al., J. Phys. A 25, 877 (1992).

[8] M. M. Guzzo and J. Bellandi, Phys. Lett. B 294, 243 (1992).

[9] A. Erdélyi, Asymptotic Expansions (Dover, New York, 1956).

[10] W. C. Haxton, Phys. Rev. Lett. 57, 1271 (1986).

[11] L. D. Landau, Phys. Z. Sowjetunion 2, 46 (1932).
[12] L. D. Landau and E. M. Lifshitz, Quantum Mechanics, Nonrelativistic Theory (Pergamon, New York, 1977).

[13] C. Zener, Proc. R. Soc. London A137, 696 (1932).

[14] S. Toshev, Phys. Lett. B 196, 170 (1987).

[15] S. T. Petcov, Phys. Lett. B 200, 373 (1988).

[16] P. I. Krastev and S. T. Petcov, Phys. Lett. B 207, 64 (1988).

[17] T. Kaneko, Prog. Theor. Phys. 78, 532 (1987).

[18] D. Notzold, Phys. Rev. D 36, 1625 (1987).

[19] T. K. Kuo and J. Pantaleone, Phys. Rev. D 39, 1930 (1989).

[20] J. Bahcall and R. K. Ulrich, Rev. Mod. Phys. 60, 298 (1988).

[21] S. Turck-Chièze et al., Astrophys. J. 335, 425 (1988).

[22] S. T. Petcov, Phys. Lett. B 191, 299 (1987).

[23] E. T. Whittaker and G. N. Watson, A Course of Modern Analysis (Cambridge University Press, Cambridge, England, 1969). 